Check for updates

Cite this: Mater. Horiz., 2019, 6,385

Received 14th September 2018 Accepted 31st October 2018

DOI: $10.1039 / \mathrm{c} 8 \mathrm{mh} 01145 \mathrm{a}$

rsc.li/materials-horizons

\section{Close-loop dynamic nanohybrids on collagen-ark with in situ gelling transformation capability for biomimetic stage-specific diabetic wound healing $\dagger$}

\author{
Zehua Liu, $\ddagger^{\mathrm{a}}$ Yunzhan Li, $\ddagger^{\text {bc }}$ Wei Li, ${ }^{* a}$ Wenhua Lian, bc Marianna Kemell, (D) d \\ Sami Hietala, (D) ${ }^{d}$ Patrícia Figueiredo, ${ }^{a}$ Li Li, ${ }^{\text {bc }}$ Ermei Mäkilä, ${ }^{e}$ Ming Ma,

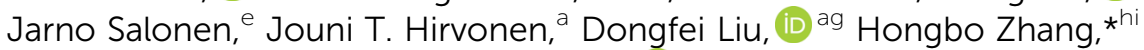 \\ Xianming Deng ${ }^{* \mathrm{bc}}$ and Hélder A. Santos (ID *ag
}

Here, an oxidation/acid dual-responsive nanohybrids/ark system was produced. The microfluidics-produced nanohybrids endow the system with an orchestrated cascade from wound detection, reactive oxygen species scavenging, drug release to hydrogel formation. The drug release behavior imitates the dynamic wound healing process, thus rendering an enhanced bio-mimetic regeneration.

Intelligent materials that adapt and respond to their environment are termed as dynamic materials, ${ }^{1}$ and have recently drawn attention for tissue engineering and regeneration. Conventional materials often fail to apprehend the dynamic and heterogeneous nature of an in vivo environment during the regeneration process. In contrast, strategically designed dynamic materials can bio-mimetically alter their physiochemical properties when encountering a specific biological irritant, further executing programmed functions, and thus holding great clinical value..$^{1-4}$

${ }^{a}$ Drug Research Program, Division of Pharmaceutical Chemistry and Technology, Faculty of Pharmacy, University of Helsinki, FI-00014, Helsinki, Finland. E-mail:wei.li@helsinki.fi, helder.santos@helsinki.fi

${ }^{b}$ State Key Laboratory of Cellular Stress Biology, Innovation Center for Cell Signaling Network, School of Life Sciences, Xiamen University, 361102, Fujian, China.E-mail: xmdeng@xmu.edu.cn

${ }^{c}$ State-Province Joint Engineering Laboratory of Targeted Drugs from Natural Products, School of Life Sciences, Xiamen University, 361102, Fujian, China

${ }^{d}$ Department of Chemistry, University of Helsinki, FI-00014, Helsinki, Finland

${ }^{e}$ Laboratory of Industrial Physics, Department of Physics, University of Turku, FI-20014, Turku, Finland

${ }^{f}$ State Key Laboratory of High Performance Ceramics and Superfine Microstructures, Shanghai Institute of Ceramics, Chinese Academy of Sciences, Shanghai 200050, China

${ }^{g}$ Helsinki Institute of Life Science (HiLIFE), University of Helsinki, FI-00014, Helsinki, Finland

${ }^{h}$ Department of Pharmaceutical Science, Åbo Akademi University, FI-20520, Turku, Finland. E-mail: hongbo.zhang@abo.fi

${ }^{i}$ Turku Center of Biotechnology, University of Turku and Åbo Akademi University, FI-20520, Turku, Finland

$\dagger$ Electronic supplementary information (ESI) available. See DOI: 10.1039/c8mh01145a

$\$$ These authors contributed to this work equally.

\section{Conceptual insights}

We have developed here a self-regulated dynamic nanohybrid that can sensitively respond to a hyperglycemic microenvironment. The nanohybrid is subsequently embedded in a collagen patch for further in vivo diabetic wound healing applications. The nanohybrid with a core/shell structure was produced through a single-step microfluidics-assisted nanoprecipitation method, where drug-loaded porous silicon (PSi) nanoparticles were encapsulated by a $\mathrm{H}_{2} \mathrm{O}_{2}$ responsive polymer, 4-(hydroxymethyl)-phenylboronic acid pinacol ester conjugated with oxidized dextran (POD). POD encapsulation can effectively block accessible pores of PSi, thus preventing a potent burst release of the drug. Upon exposure to high levels of glucose, GOx can initiate glucose oxidation, generating gluconic acid and $\mathrm{H}_{2} \mathrm{O}_{2}$. Then, POD can consume the produced $\mathrm{H}_{2} \mathrm{O}_{2}$ to undergo degradation and further release the encapsulated drug. The degradation product of POD, oxidized dextran (ODEX), can in situ form a hydrogel with the collagen patch. This in situ nanoparticle/hydrogel transformation creates a moist environment around the wound bed, altering the stiffness of the patch to better mimic elastic properties of the natural soft tissues. Glucose content within the wound bed also gradually decreased as a result of less bleeding and interstitial fluid exudation, thus a stage/temporal-specific drug release can also be achieved.

Diabetic foot ulcer (DFU) is a critical non-healing complication for diabetic patients. ${ }^{5}$ Over the past decades, great efforts have been made to overcome this challenge. However, despite the reporting of several bioactive substances (e.g., small molecule drugs, growth factors, and cytokines) used to accelerate the healing process, the combination of a "smart" delivery system and tailored release behavior is equally indispensable. As for tissue engineering, long-term or un-regulated drug delivery may cause aberrant regeneration and even formation of carcinoma-like structure. $^{6,7}$ Inspired by the meticulous self-regulating system within the human body, where regenerative enhancement is dynamically altered during different healing stages, ${ }^{8-10}$ prospective materials for fabricating the envisioned bio-mimetic delivery system should be able to sense and respond to the healing process, and concurrently alter their drug releasing behavior. 
Phenylboronic acid ester (PAE) is an important building block for constructing a series of oxidation responsive materials. ${ }^{11-14}$ This unique feature renders it suitable for application in chronic wounds due to elevated concentrations of common reactive oxygen species (ROS) within the wound bed. ${ }^{11}$ However, despite PAE's ROS scavenging profile and boronate degradation products, which favor augmentation of the wound healing rate, few papers have applied PAE based materials for DFU caring. ${ }^{15,16}$ This may be explained by selective oxidation behavior of PAE, which can only be oxidized by $\mathrm{H}_{2} \mathrm{O}_{2}$ and not by other ROS, such as superoxide, hypochlorous acid or hydroxyl radical ${ }^{17,18}$ resulting in a sensitivity concern when applied in vivo. The low solubility of PAE containing polymers in various solvents further constrains the productions of its electrospun nanofibrous membrane or hydrogels. ${ }^{13}$ Therefore, a more deliberately designed system should be conceived for further applications.

High level of glucose is another pivotal factor in diabetes and may impact the delivery system construction for DFU repair. Among the reported glucose responsive materials so far, the introduction of glucose oxidase (GOx) is especially advantageous for diabetic wound healing, as it can effectively decrease the glucose level that is likely of great importance for diabetic wound healing management. ${ }^{19,20}$ However, GOx-containing bulk hydrogels exhibit an insensitive response to glucose. ${ }^{21,22}$ GOx-Containing membranes suffer from low mechanical strength, which can result in premature leakage of the drug. ${ }^{21,22}$ Moreover, the side products, especially of hydrogen peroxide that is generated by this oxidation process, may further increase concerns of its biocompatibility. ${ }^{23,24} \mathrm{~A}$ viable approach to circumvent this issue is to fabricate a closed-loop nanocarrier that can expend its side products, and sequentially generate the required downstream actions.

Based on above considerations, herein, we have developed a self-regulated dynamic nanohybrid that could sensitively respond to the hyperglycaemic microenvironment. The nanohybrid with a core/shell structure was produced through a single-step microfluidics-assisted nano-precipitation method, ${ }^{25,26}$ where drug-loaded porous silicon (PSi) nanoparticles were encapsulated by a $\mathrm{H}_{2} \mathrm{O}_{2}$ /acid dual-responsive polymer formed by 4-(hydroxymethyl)-phenylboronic acid pinacol ester conjugated with oxidized dextran (POD). PSi has been widely investigated for biomedical applications due to its biocompatibility and flexibility in loading different types of cargos. ${ }^{27,28}$ Moreover, its main degradation product, orthosilicic acid, has also been known to favor the cutaneous traumatic healing, ${ }^{29-31}$ thus making PSi an attractive candidate for DFU application. POD encapsulation can effectively block accessible pores of PSi, thus preventing a potent burst release of the drug. Furthermore, the redundant aldehyde groups on the outer surface of POD can both immobilize GOx through the formation of a pH-responsive dynamic covalent bond (imine), ${ }^{32,33}$ and/or simultaneously anchor other amine containing drugs. The benefits of such a delivery system arise from the interaction between the oxidation/ acid dual-responsive POD and GOx, which confers control over the drug release property in a glucose concentration dependent manner. To facilitate in vivo application, the nanohybrids were feasibly loaded onto a collagen ark. Interestingly, the degradation product of POD, oxidized dextran (ODEX), could in situ form a hydrogel with the collagen patch. This in situ nanoparticle/hydrogel transformation can create a moisture environment around the wound bed, altering the stiffness of the patch to better mimic the elastic properties of natural soft tissues. ${ }^{34,35}$ More importantly, along with dermis regeneration and cutaneous gap closure during the treatment, the glucose content within the wound bed will gradually decrease as a result of less bleeding and interstitial fluid exudation - thus, a stage/temporal-specific drug release schedule can be achieved. ${ }^{36}$ Herein, the physicochemical properties of the nanohybrids were characterized, and the glucose triggered orchestrated cascade and its potent bioactivity was evaluated both in vitro and in vivo through diabetic wound mice model.

Fig. 1A schematically illustrates the fabrication and dynamic transformation of the nanohybrids during the treatment process. We modified oxidized dextran (ODEX) with phenylboronic pinacol ester to produce POD. The synthesis procedure and its degradation under $\mathrm{H}_{2} \mathrm{O}_{2}$ irritation is briefly described in Fig. S1 (ESI†). Successful oxidation of dextran was first confirmed by fourier transform infrared spectroscopy (FTIR), as a new band was observed at $1720 \mathrm{~cm}^{-1}$, which was attributed to the aldehyde groups (Fig. S2, ESI $\dagger$ ). ${ }^{37}$ Oxidation degree was further determined as $40.2 \%$ by ${ }^{1} \mathrm{H}$ NMR by the carbazone formation method (ESI $\dagger$ ). 4-(Hydroxymethyl)phenylboronic acid pinacol ester (PAPE) was then conjugated to the residue hydroxyl groups of oxidized dextran via a carbonate linkage. The substitution degree of hydroxyl groups was $c a .31 \%$, as determined from the ${ }^{1} \mathrm{H}$ NMR peak area with the peak of dextran used as the standard (Fig. S3, ESI $\dagger$ ). ${ }^{13}$ The conjugation of PAPE enhanced the hydrophobicity of polymer, as the new product precipitated out from an aqueous solution. A microfluidic assisted nanoprecipitation method was used to construct PSi@POD, and the nanoprecipitation process was triggered by a passive microfluidic mixing in a co-flow glass capillary microtube. ${ }^{25,38}$ The outer POD layer within the microtube was formed through nucleation and nanoprecipitation driven by diffusion and mixing between the solvent (EtOH/ dd $\left.-\mathrm{H}_{2} \mathrm{O}=8: 2, \mathrm{v} / \mathrm{v}\right)$ and anti-solvent $\left(\mathrm{dd}-\mathrm{H}_{2} \mathrm{O}\right)$ streams across the interface. It should be noted that no extra surfactant was needed to obtain particles with satisfactory polydispersity index (PdI) during this process, as the unmodified hydroxyl groups on ODEX function as stabilizers. ${ }^{39}$ Transmission electron microscopy (TEM) images indicated the successful encapsulation of PSi nanoparticles (Fig. 1B(a)) in the POD matrix. As shown in Fig. $1 \mathrm{~B}(\mathrm{~b})$, after encapsulation, the irregular shape and the porous structure of PSi were fully covered and new nanohybrids with spherical morphology were formed. Energy dispersive X-ray microscopy (EDX) was carried out to further confirm the successful encapsulation (Fig. 1B(c)). At physiological conditions, free aldehyde groups on the surface steadily formed Schiff-bases with primary amine groups, ${ }^{40}$ which were further utilized for surface immobilization of both GOx and deferoxamine (DFO) without causing obvious morphology changes (as observed from TEM) (Fig. 1B(d)). Scanning electron microscopy (SEM) 

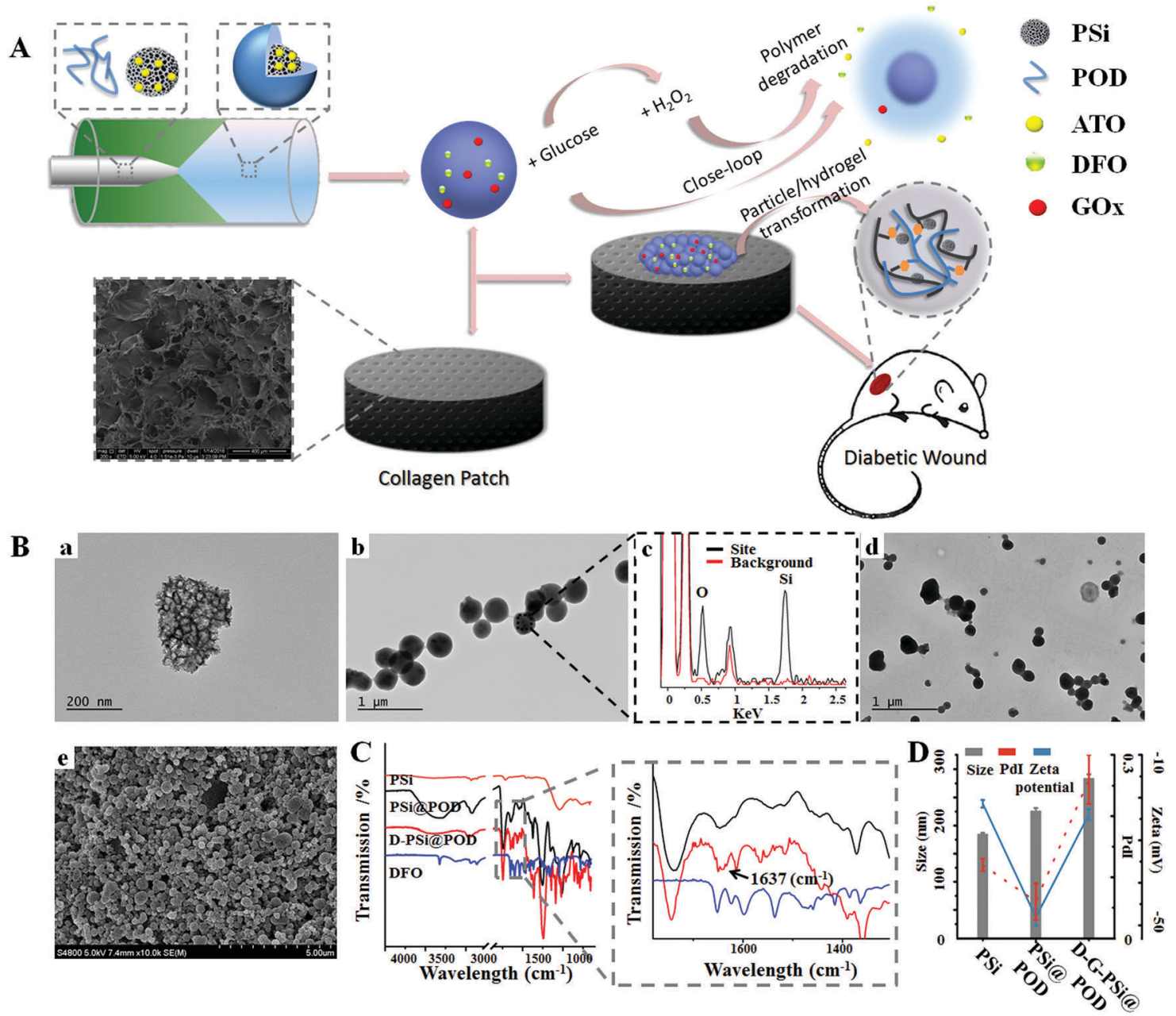

Fig. 1 Preparation and characterization of DFO, GOx conjugated, ATO loaded nanohybrid (D-G-PSi@POD). (A) Schematic illustration of the construction of glucose responsive delivery system and the orchestrated cascade for diabetic wound care. The detrimental side product of $\mathrm{H}_{2} \mathrm{O}_{2}$, was utilized and consumed, further aiding the close-loop system. (B) TEM/SEM images showing morphologies of (a) PSi, (b) PSiaPOD and (c) their respective EDX spectra at particle site and the background, (d) D-G-PSi@POD and (e) D-G-PSi@POD embedded on the collagen patch without obvious morphology changes. (C) FTIR spectra of PSi, PSi@POD, D-PSi@POD and DFO, suggesting a successful encapsulation and Schiff-base formation. (D) Size, Pdl and zeta-potential shift during each step of synthesis, suggesting a successful modification in each step.

images of the prepared particles embedded in the collagen patch illustrated a uniformly formed nano-network (Fig. 1B(e)), confirming it to be a facile method for the preparation of a topical patch. The successful encapsulation and Schiff-base formation were verified by FTIR (Fig. 1C). After encapsulation, the band at $1045 \mathrm{~cm}^{-1}$ for $\mathrm{Si}-\mathrm{O}$, which is a typical band for PSi, disappeared, and was replaced by the band at $1010 \mathrm{~cm}^{-1}$ due to polysaccharide encapsulation. The typical band from Schiff-base $(\mathrm{C}=\mathrm{N})$ was observed at $1637 \mathrm{~cm}^{-1}$ after DFO conjugation. ${ }^{25,41}$ After the encapsulation, the particle size changed from $185 \pm 2$ (PSi) to $226 \pm 5 \mathrm{~nm}$ (PdI: $0.12 \pm 0.01$ vs. $0.06 \pm 0.03$ )

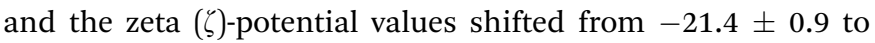
$-48.1 \pm 2.0 \mathrm{mV}$, while after Schiff-base formation both size $(284 \pm 7)$ and PdI $(0.26 \pm 0.04)$ increased and the zeta potential changed to $-23.9 \pm 1.2 \mathrm{mV}$ due to the surface drug/protein immobilization (Fig. 1D).

The introduction of GOx in the nanohybrids can endow the system with an ability to respond to different glucose levels, as glucose can be catalyzed by GOx to generate gluconic acid and hydrogen peroxide. ${ }^{42}$ The glucose-dependent $\mathrm{H}_{2} \mathrm{O}_{2}$ generation and $\mathrm{pH}$-drop were separately confirmed through the $2^{\prime}, 7^{\prime}$ dichlorodihydrofluorescein diacetate (DCFH-DA) and phenolred assisted colorimetric methods (Fig. S4, ESI $\dagger$ ). It was noted that at glucose concentration of $4 \mathrm{mg} \mathrm{mL}^{-1}$, which is the typical glucose level at hyperglycemia, the $\mathrm{H}_{2} \mathrm{O}_{2}$ yield was rather high $(380 \pm 7 \mu \mathrm{M})$. This concentration may bring potent damage to the surrounding environment when applied for biomedical applications. We hypothesized that the co-existing POD could consume $\mathrm{H}_{2} \mathrm{O}_{2}$ and initiate the downstream action, therefore $\mathrm{H}_{2} \mathrm{O}_{2}$ induced degradation behavior of PAPE-ODEX was further investigated. Macroscopic images confirmed the $\mathrm{H}_{2} \mathrm{O}_{2}$ triggered polymer degradation (Fig. 2A(a)). Bare POD particles were suspended in $\mathrm{H}_{2} \mathrm{O}$ with different concentrations of $\mathrm{H}_{2} \mathrm{O}_{2}$ and the degradation process was observed by measuring light scattering at $500 \mathrm{~nm}$ (Fig. 2A(b)). The results suggested a highly sensitive $\mathrm{H}_{2} \mathrm{O}_{2}$ concentration-dependent degradation of POD 
A

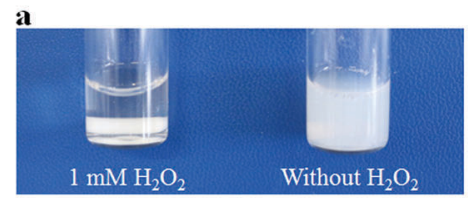

b

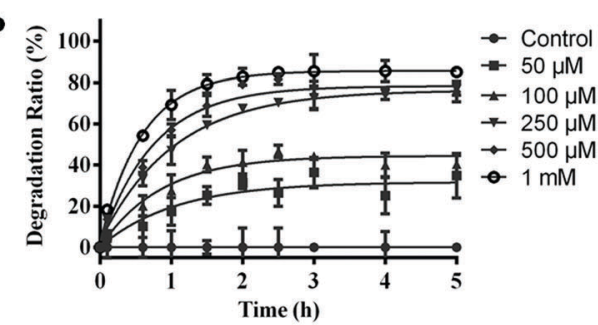

c

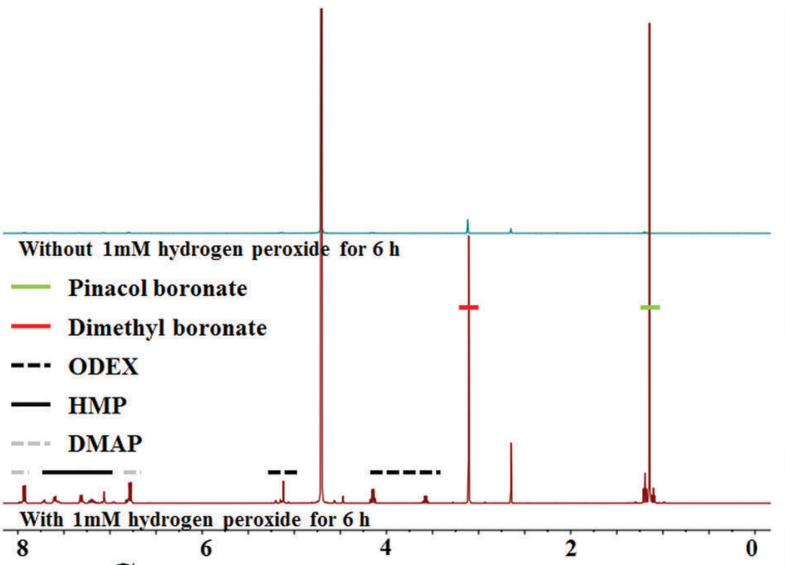

B

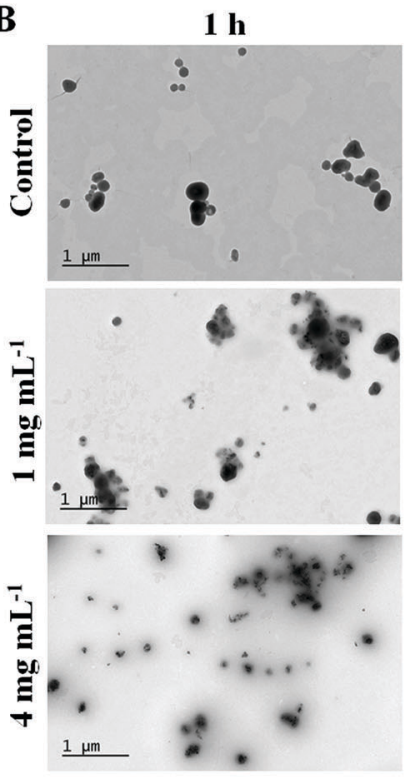

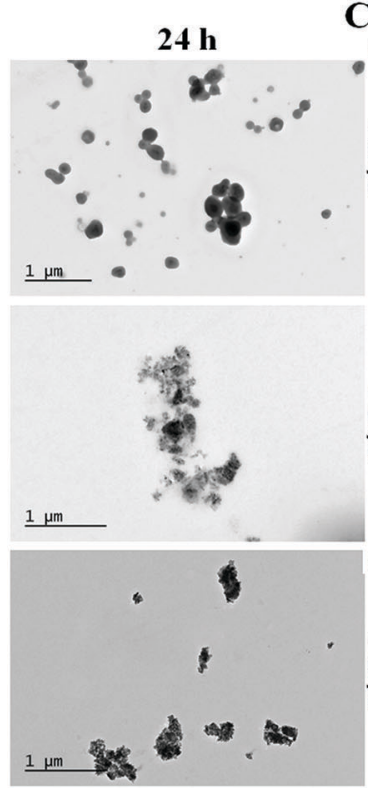

C

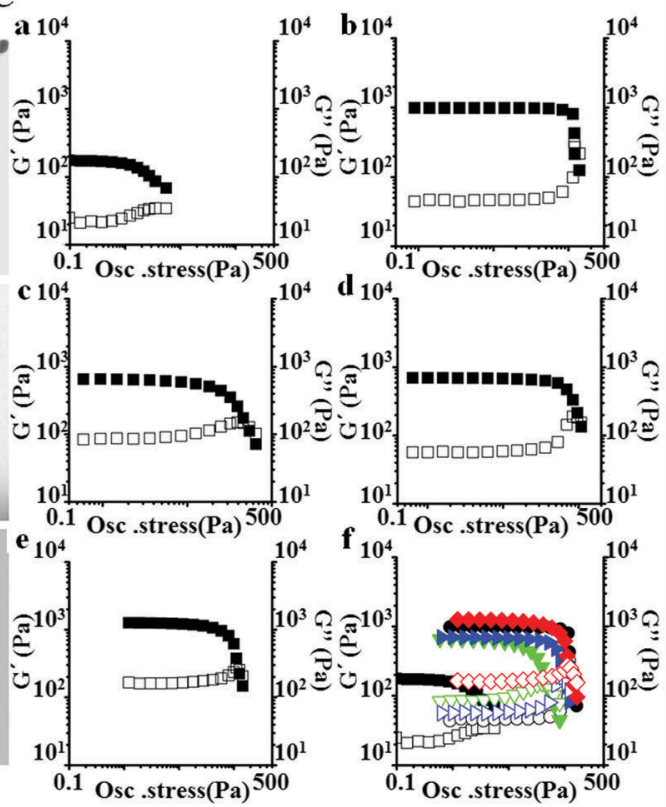

Fig. 2 (A) Investigation of the degradation of POD including (a) macroscopic images, showing $\mathrm{H}_{2} \mathrm{O}_{2}$ induced POD degradation; (b) quantitative investigation of degradation behavior via colorimetric method; (c) ${ }^{1} \mathrm{H}$ NMR spectrum of POD in $\mathrm{D}_{2} \mathrm{O}$ with or without addition of $\mathrm{H}_{2} \mathrm{O}_{2}$ confirmed the degradation behavior of POD. (B) TEM images of D-G-PSi@POD with different concentrations of glucose at different time-points, suggesting the glucose dependent disassembly of the nanohybrid. (C) Mechanical characterization of different hydrogels showing the in situ nanoparticle/hydrogel transformation. Rheological dynamic oscillatory stress sweep tests of (a) C, (b) COD, (c) CP, (d) CP1, (e) CP4 and (f) the stacking figure. Solid data points in a-f correspond to the storage modulus $G^{\prime}$. Open data points in a- $f$ correspond to the loss modulus $G^{\prime \prime}$. In $f$, black square, black circle, green down-triangle, blue right-triangle and red diamond respectively correspond to C, COD, CP, CP1 and CP4.

and the mechanism was similar to those reported elsewhere. ${ }^{13}$ Briefly, POD consumes $\mathrm{H}_{2} \mathrm{O}_{2}$ and degrades into ODEX, p-hydroxymethylphenol (HMP), pinacol boronate and dimethyl boronate, as confirmed by ${ }^{1} \mathrm{H}$ NMR (Fig. 2A(c)). TEM images further confirmed this close-loop cascade (Fig. 2B). An integrated PSi@POD structure could be observed after $24 \mathrm{~h}$ incubation without the addition of glucose. However, after $1 \mathrm{~h}$ of incubation at $1 \mathrm{mg} \mathrm{mL}^{-1}$ glucose concentration, the edge of the particle became obscure upon disassembly of the nanohybrid, as a result of $\mathrm{H}_{2} \mathrm{O}_{2}$ induced POD degradation. This phenomenon was more evident upon increasing the glucose concentration to $4 \mathrm{mg} \mathrm{mL}{ }^{-1}$. After $24 \mathrm{~h}$, the shell structure of POD was barely visible at $4 \mathrm{mg} \mathrm{mL}^{-1}$ glucose concentration and at $1 \mathrm{mg} \mathrm{mL} \mathrm{m}^{-1}$, only an indistinct polymeric layer was found.
Overall, these results suggested a glucose induced close-loop particle degradation cascade.

To better facilitate the in vivo application of the nanohybrid, we further embedded the particles into a collagen scaffold. Collagen has shown good attachment/adhesion to different type of cells and has been widely applied in wound care applications. ${ }^{43}$ However, bare collagen hydrogel usually results in poor stability and mechanical properties, which may restrict the wound closure efficacy. ${ }^{44,45}$ The degradation product of POD (ODEX) has large number of aldehyde groups, thus it may function as a natural cross-linker during the in situ hydrogel formation. ${ }^{44}$ To confirm our hypothesis, SEM images of lyophilized hydrogel were first obtained. After full dispersion and lyophilization, the morphologies of bare and particle 
embedded collagen hydrogels were observed to be different: The pores of the latter hydrogel were smaller (Fig. S5, ESI $\dagger$ ), which is favorable for improving the strength of hydrogel. The oscillatory stress sweep tests (Fig. 2C) and angular frequency sweep tests (Fig. S6, ESI $\dagger$ ) of different hydrogels, including bare collagen (C), collagen + ODEX (COD), collagen + PSi@POD (containing the same concentration of ODEX, CP), collagen + PSi@POD + $1 \mathrm{mg} \mathrm{mL}^{-1}$ glucose (CP1) and collagen + PSi@POD + $4 \mathrm{mg} \mathrm{mL} \mathrm{m}^{-1}$ glucose (CP4) were performed and the corresponding storage moduli $\left(G^{\prime}\right)$ and loss moduli $\left(G^{\prime \prime}\right)$ were recorded. Viscoelastic properties of the different hydrogels were cumulatively characterized by the viscoelastic figure of merit (VFOM), calculated as VFOM $=\left|G^{*}\right| \tan \delta$, where $G^{*}=\left(G^{\prime 2}+G^{\prime 2}\right)^{0.5}$ and $\tan \delta=G^{\prime \prime} / G^{\prime} .^{46}$ Fig. 2C clearly suggests a structural breakdown occurring at higher shear stress when adding ODEX or PSi@POD, consequently, the CP4 group showed the highest stiffness enhancement. Similar tendency was also observed from VFOM value, where CP4 gave a value of $1668 \mathrm{~Pa}$ at $8 \mathrm{~Hz}$ compared to $322 \mathrm{~Pa}$ of C (Table S1, ESI $\dagger$ ). The reason for this enhanced viscoelastic property of $\mathrm{CP} 4$ compared to COD, despite the same cross-linker concentration, is related to the decreased local $\mathrm{pH}$ value caused by the synchronously produced gluconic acid. It has been previously suggested that insoluble collagen charges will become positive within an acidic environment, allowing the collagen fibers to repel each other and de-aggregate - further facilitating the interactions between collagen and the cross-linker, ${ }^{47}$ and imparting a higher structural stiffness for the CP4 group.
We further investigated the drug loading and release profile of D-G-PSi@POD in vitro. In this study, small molecule drugs are chosen as model drugs since they are more attractive for their lower product development costs and higher commercialization potential. Thus, both hydrophobic (atorvastatin, ATO) and hydrophilic (DFO) drugs were loaded in the nanohybrid. Topical delivery of DFO can accelerate angiogenesis by promoting the expression of hypoxia inducible factor $1 \alpha($ Hif- $1 \alpha)$ and vascular endothelial growth factor (VEGF), which further promote the wound healing process $;^{48,49}$ while the positive effect of ATO and DFU due to their anti-inflammatory effect has been previously reported. ${ }^{50,51}$ Herein, the hydrophobic drug ATO was loaded into PSi, because PSi has displayed improvement of the in vivo drug bioavailability, especially for hydrophobic drugs. ${ }^{52}$ The hydrophilic drug DFO was anchored on the outer surface of the nanohybrid through dynamic covalent bond during Schiff-base formation. The final loading degrees of ATO and DFO were $2.4 \pm 0.2 \%$ and $4.7 \pm 0.5 \%$, respectively. Due to the coverage of POD layer, ATO showed a clear $\mathrm{H}_{2} \mathrm{O}_{2}$ concentration dependent release. However, maximized ATO release was achieved at relatively high $\mathrm{H}_{2} \mathrm{O}_{2}$ concentration, while at common physiological conditions the drug release was barely observed (Fig. S7A, ESI $\dagger$ ). As illustrated above, the introduction of GOx may not only solve this problem, but the synchronously generated gluconic acid can also decrease the surrounding $\mathrm{pH}$ value and trigger the release of DFO. The ATO/DFO release behavior from both free particles (Fig. S7B and $\mathrm{C}, \mathrm{ESI} \dagger$ ) and particles loaded collagen patch (Fig. 3A) were
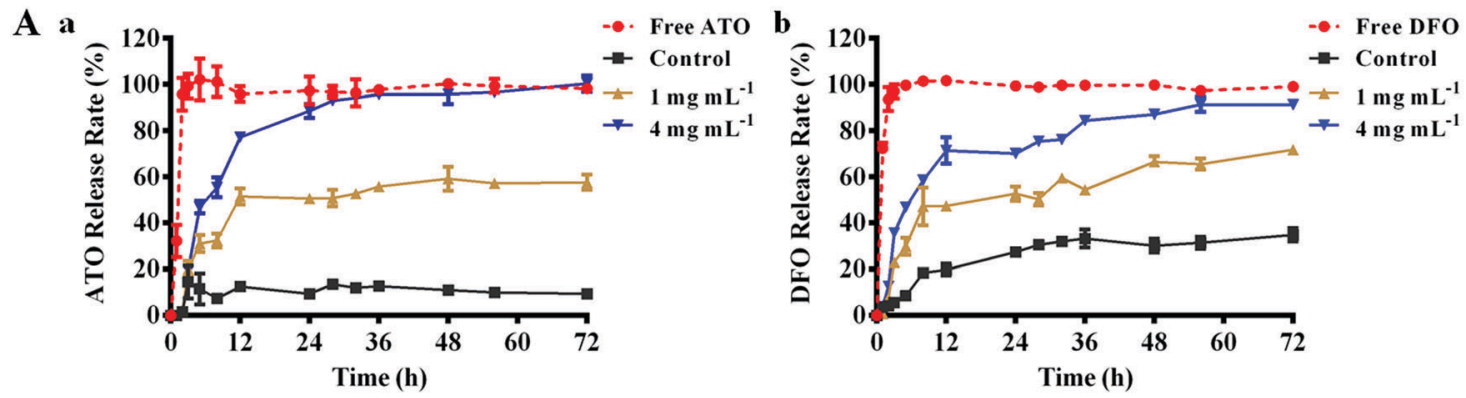

B a
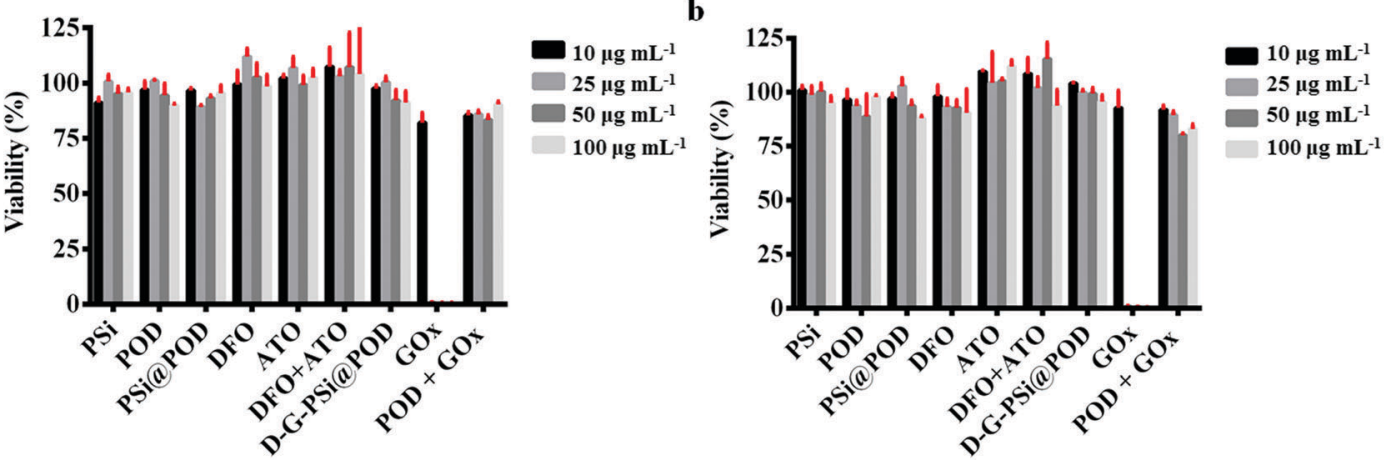

Fig. 3 (A) Transwell assisted in vitro (a) ATO and (b) DFO release curves for different formulations embedded on collagen patches at different glucose concentrations $\left(0,1\right.$ or $4 \mathrm{mg} \mathrm{mL}^{-1}$ ) in phosphate buffer saline (PBS) within $72 \mathrm{~h}$. (B) Biocompatibility studies of different components. Cell viability of fibroblast cells at $24 \mathrm{~h} \mathrm{(a)} \mathrm{and} 48 \mathrm{~h}$ (b). Different components with series of concentrations were incubated with fibroblast cells for $24 \mathrm{~h}$ (a) and $48 \mathrm{~h}$ (b). Concentrations indicate the amount of PSi within the system or the corresponding concentrations in D-G-PSi@POD with constant PSi concentration. Data are shown as mean \pm SD $(n \geq 3)$. 
investigated at different glucose concentrations $(0,1$ and $4 \mathrm{mg} \mathrm{mL}^{-1}$ ). Sustained and glucose-triggered ATO/DFO releases were observed when the nanohybrid was employed. Only $9.5 \pm 1.4 \%$ of ATO and $35 \pm 2.9 \%$ of DFO were released after $72 \mathrm{~h}$ without glucose addition, whereas for free drugs loaded collagen patch, over $97 \%$ of the drugs were released within the first $3 \mathrm{~h}$. Cumulative ATO/DFO release was significantly higher when increasing the glucose concentration, wherein after $72 \mathrm{~h}$, the cumulative release of ATO/DFO was $57 \pm 2.7 \%$ and $71 \pm 22 \%$, respectively, at $1 \mathrm{mg} \mathrm{mL}^{-1}$ glucose concentration and this value increased to more than $90 \%$ at glucose concentration of $4 \mathrm{mg} \mathrm{mL}^{-1}$. This glucose dependent release may further lead to stage specific, temporal drug release from the patch. This is important as the local glucose content in diabetic wounds is highly correlated to the diabetes condition and tissue repairing process. ${ }^{36}$ With the closure of the dermis gap, fewer blood and tissue exudates can infiltrate to the wound bed, thus altering the release behavior of both drugs to benefit the therapeutic efficiency and rehabilitation.

Biocompatibility of the different components in the nanosystem was further evaluated using fibroblast cells. After 24 and $48 \mathrm{~h}$ co-incubation, most of the components did not cause an obvious decrease in cellular viability, preliminarily indicating low cytotoxicity of the drugs and the materials (Fig. 3B). Noticeably, a sharp cell viability decrease was observed for the GOx group (decreased to $1 \%$ ), due to the generation of $\mathrm{H}_{2} \mathrm{O}_{2}$ upon addition of GOx to the high glucose DMEM medium $\left(4 \mathrm{mg} \mathrm{mL}^{-1}\right.$ glucose). However, POD particles conjugated with GOx showed a totally reversed effect on the cytotoxicity with a cell viability of $83 \pm 2.0 \%$ at the highest concentration ( $48 \mathrm{~h}$ ), suggesting in situ close-loop $\mathrm{H}_{2} \mathrm{O}_{2}$ consumption.

In vitro angiogenesis was also evaluated on human umbilical vascular cells (HUVECs) through a typical transwell facilitated tube-formation assay. It has been previously suggested that the angiogenesis-promoting efficacy of DFO can interfere with the activity of prolylhydroxylases (PHDs), which is a key factor in the degradation process of Hif- $1 \alpha$. This excess degradation of Hif- $1 \alpha$ will further promote the downstream vascular endothelial growth factor (VEGF) expression, thus activating the angiogenesis process. ${ }^{49}$ As can be seen in Fig. S8 (ESI $\dagger$ ), the HUVEC cells merely showed tubulization without the addition of drugs after $12 \mathrm{~h}$. The tube-formation efficacy of free drugs and D-G-PSi@POD was further compared, and the HUVEC cells cultured with free drugs were observed to show more vascular tube formation compared with those cultured with D-G-PSi@POD at $6 \mathrm{~h}$. However, after $12 \mathrm{~h}$, the HUVEC cells displayed similar sprouting when administrated with free drugs, whereas for D-G-PSi@POD group, longer tubules were subsequently observed. This suggests a delayed yet sustained drug release from the nanohybrid, thus confirming the in vitro drug release.

Streptozotocin (STZ)-induced diabetic mice with full thickness wounds were used to evaluate the in vivo performance of our system for diabetic wound closure. The mice were randomly divided into four groups $(n=5)$ and topically administrated with different samples, including (1) blank PSi@POD embedded in collagen patch (CP), (2) free agents (ATO, DFO and GOx) embedded in collagen patch (CF), (3) ATO and GOx loaded PSi@POD embedded in collagen patch (PA), and (4) ATO, DFO and GOx loaded PSi@POD embedded in collagen patch (PAD). The administered dosage can be feasibly tuned by altering the amount of particles within the collagen patch. Herein, we used $200 \mu \mathrm{g}$ (corresponding to the amount of PSi) per patch for the in vivo study, changing the patch every 3 days. A sharp debridement, a standardized protocol for diabetic wound management, ${ }^{53}$ was conducted before administrating a new patch.

Images of wounds on the animals were taken at different time intervals and the wound area was quantified by tracing its margins and calculating percentage of new area to the original wound size, using ImageJ software. As can be seen in Fig. 4A and Fig. S9 (ESI $\dagger$ ), significant lessening of wound area was observed during the first $3 \mathrm{~d}$ for the PAD group, and a statistical difference between PAD and PA suggests the positive effect of DFO. It has previously been suggested that the angiogenesis amelioration effect of DFO, via the activation of vascular endothelial cell function, and early stage angiogenesis are key factors for accelerating the healing process. ${ }^{35,49}$ Moreover, the recovery rate was also significantly faster for PAD compared to free drugs group (CF), which may be attributed to the sustained release behavior of the system, as shown above. At day 6, the wound closing ratio for PAD was $70 \pm 4.7 \%$ compared to $52 \pm 7.2 \%$ for the CF group $(P<0.01)$ and $44 \pm 3.9 \%(P<0.01)$ for the PA group. However, after the 6th day, the healing rate of PAD group was highly impaired, whereas the closing rate of wound area of the CF groups became faster. The retarded healing process may be caused by the dermis development, and thus, less tissue exudate and bleeding limited the release of drugs from the nanosystem, whereas the release of free drugs was not affected.

To confirm our hypothesis, full scanning hematoxylin-eosin (H\&E) staining images of different groups at day 7 (Fig. 4B) and day 14 (Fig. S10, ESI $\dagger$ ) were obtained and analyzed. Crosssectional analysis of histological wound sections allowed detection of the crawling distance of new skin tissues. Both CF and PAD showed considerable wound closure compared to the PA and CP groups. However, more obvious epidermis regeneration was observed for the PAD group, suggesting the advanced healing stage of PAD group compared to the free drugs group. The epidermis functions as barrier to regulate the fluid content around the wound bed, and a more intact epidermis can restrain the blood and tissue exudate infiltration, thus, the release of drug was thereby correlated to the healing stage.

Immunofluorescence staining for CD31 was studied to investigate the distribution of microvessels in the regenerated dermis near the wound bed, and the microvessel density (MVD) was semi-quantitatively evaluated using an established protocol. ${ }^{35}$ As shown in Fig. 4C, CD31 staining was significantly increased in the dermis treated PAD $(181 \pm 33$ microvessels/hot spot) compared to the CP ( $30 \pm 6$ microvessels/hot spot), PA (56 \pm 31 microvessels/hot spot) and CF (128 \pm 28 microvessels/ hot spot) groups at day 7 (Fig. S11, ESI $\dagger$ ). An overall subsided MVD was observed at $14 \mathrm{~d}$ at the final healing stage. , $^{8,9,54}$ However, the MVD of CF was only moderately reduced, whereas 
A
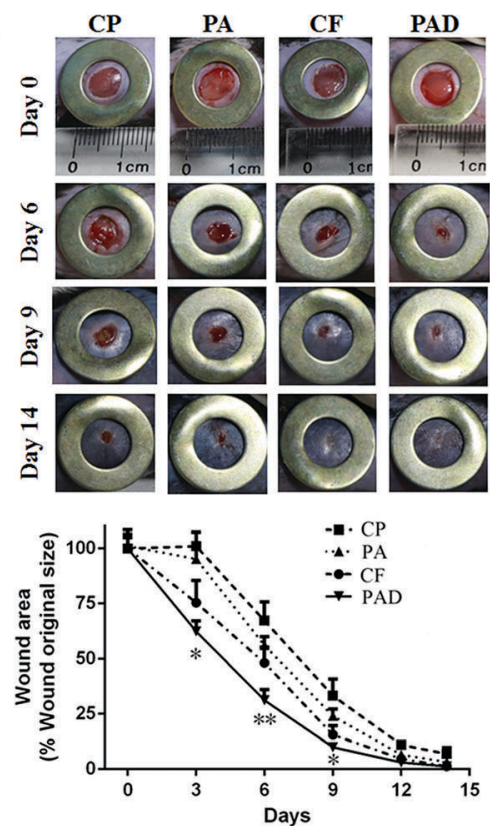

B

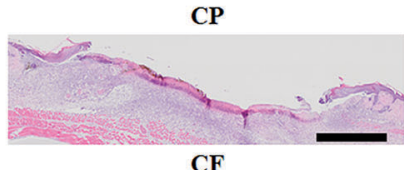

CF

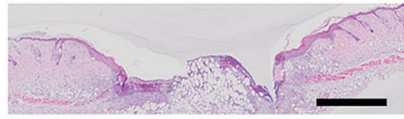

C

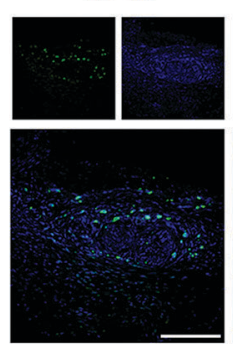

CP-14d

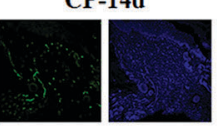

14 days

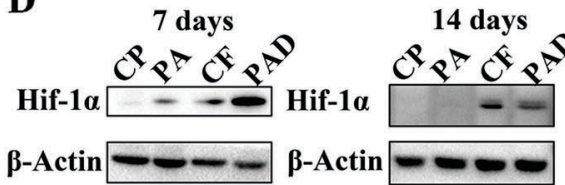

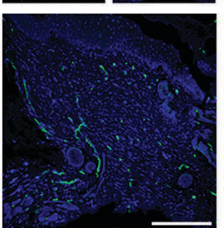

PA-7d

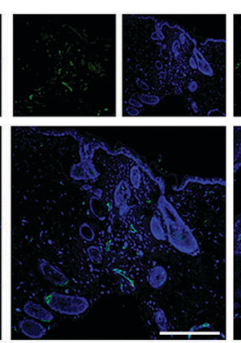

PA-14d
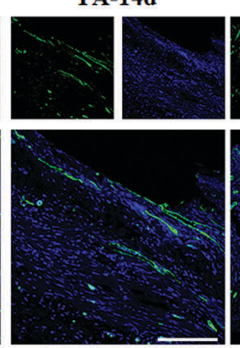

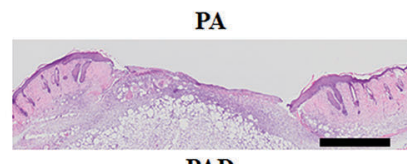

PAD

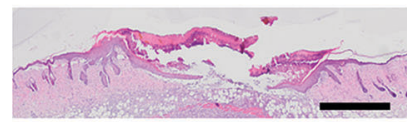

CF-7d

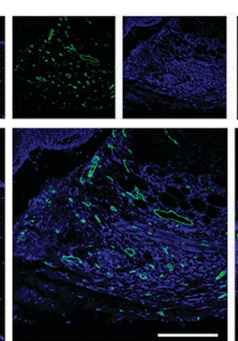

CF-14d
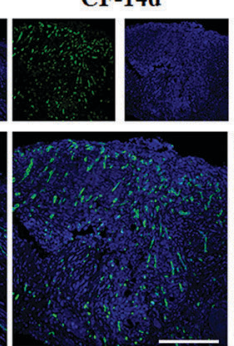

PAD-7d

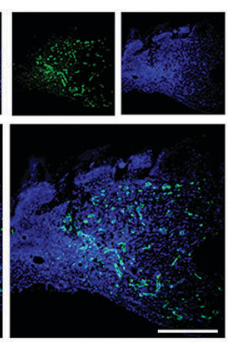

PAD-14d
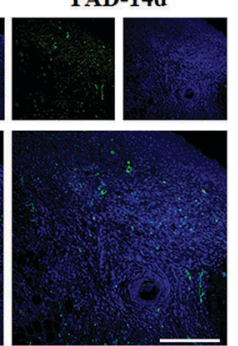
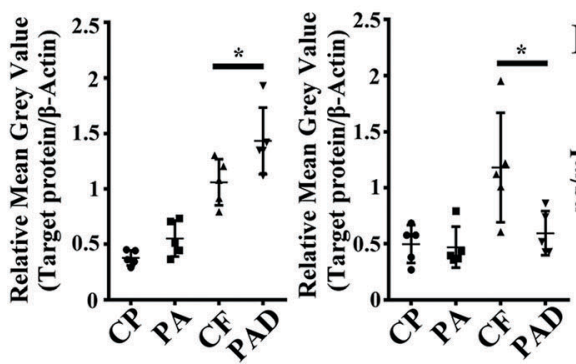

E
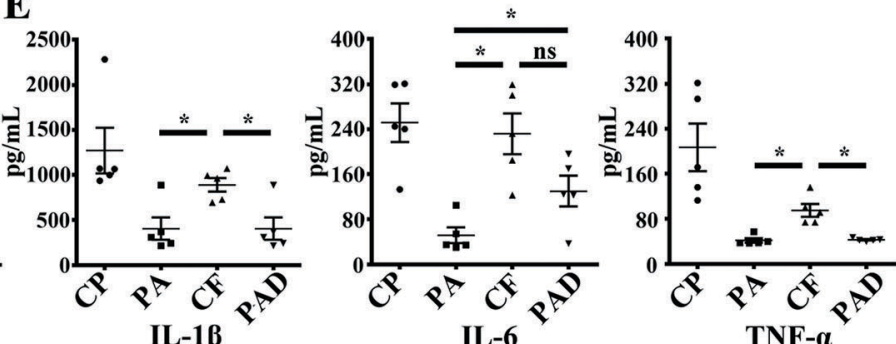

Fig. 4 (A) Digital photographs of the wounds at days 0, 6, 9, and 14 after injury, along with quantification of the wound area using ImageJ software. Error bars in (A) indicate the SD value $(n=5)$. ${ }^{*}<0.05$ and ${ }^{* *} P<0.01$. (B) H\&E stained full-thickness wound beds on days 7 suggesting the different recovery degrees of different groups and less exudate caused by potent epidermis regeneration. Scale bars are $1 \mathrm{~mm}$. (C) Immunofluorescence staining of CD31 suggesting the different angiogenesis degrees of different groups, showing the superior treatment efficacy of D-G-PSi@POD compared to the free drug at an early stage (7 d) and the impaired augmented angiogenesis at $14 \mathrm{~d}$ due to the stage-specific drug release. All scale bars are $200 \mu \mathrm{m}$. (D) Western blot analysis of Hif- $1 \alpha$ at different healing stages, suggesting a mechanism for the treatment regimen and the stage-specific regeneration enhancement. Data are shown as mean $\pm \mathrm{SD}(n=5)$, ${ }^{\star} P<0.05$. (E) Quantitative analysis of 3 major pro-inflammatory cytokines (IL-1 $\beta$, IL-6, and TNF- $\alpha$ ) within the wound bed at day 7, suggesting the advantage of co-delivery strategy using single particle. Data are shown as mean $\pm \operatorname{SEM}(n=5)$, * $P<0.05$.

the endothelial regression for PAD was more obvious, resulting in a higher MVD of CF compared to PAD $(124 \pm 25$ vs. $69 \pm 25$ microvessels/hot spot, respectively, ${ }^{*} P<0.05$, Fig. S11, ESI $\left.\dagger\right)$. Similar tendency was also observed from immunohistochemistry analysis of the fraction of Ki-67 expressing cells within the granulation tissue, which indicates the existence of cellular proliferation within the wound (Fig. S12, ESI $\dagger$ ). Western blot analysis also semi-quantitatively confirmed this stage specific regeneration enhancement: For day 7 , the Hif- $1 \alpha$ expression of PAD group was higher than that of $\mathrm{CF}$, but a reversed phenomenon was observed at day 14 (Fig. 4D and Fig. S13, ESI $\dagger$ ). Consequently, the production of VEGF exhibited a similar tendency (Fig. S14, ESI $\dagger$ ). ${ }^{55}$ Stage specific controlled drug release is a crucial factor in regenerative medicine. For example, topical administration of VEGF can significantly enhance the wound healing process in diabetic mice, however, it has been shown that the long-term expression or administration of VEGF in both ischemic and non-ischemic tissues may cause aberrant vessels formation and even angioma-like vascular tumors. ${ }^{56,57}$ The application of our nanosystem can mimic the healing stage by monitoring the implicated factor, further altering the release content accordingly, and thereby enhancing the treatment.

One advantage of applying nanoparticles as a delivery system is their feasibility in achieving combination therapy by 
simply co-loading multiple therapeutic agents into a single carrier. However, simultaneous loading of therapeutics with different physicochemical properties can present challenges. Herein, both hydrophobic ATO and hydrophilic DFO were co-loaded in PSi@POD. Despite the positive effect of DFO on angiogenesis, it was also been shown to stimulate inflammation, however, the combination application of statins may retard this phenomenon. ${ }^{58-60}$ To evaluate the inflammation status of different groups, the secretion of three major pro-inflammatory cytokines (interleukin $1 \beta$ (IL-1 $\beta$ ), interleukin 6 (IL-6) and tumor necrosis factor $\alpha($ TNF- $\alpha)$ ) within wound bed were evaluated at day 7 (Fig. 4E) and day 14 (Fig. S15, ESI $\dagger$ ). Both particles involved groups (PA, PAD) showed significantly lower inflammation response compared to free drugs group (CF), as the generated $\mathrm{H}_{2} \mathrm{O}_{2}$ was effectively consumed by POD. Furthermore, in spite of different rehabilitation degrees of $\mathrm{PA}$ and $\mathrm{PAD}$, the inflammation status of PA was equal to or even superior than $\mathrm{PAD}$, suggesting the positive effect of ATO and the rationale of treatment regime.

\section{Conclusions}

In summary, due to the simple design and application of a PAE containing oxidation responsive polymer, we have successfully prepared a nanohybrid embedded collagen patch for diabetic wound healing. The feasible combination of nanohybrid and collagen patch integrated both the sensitive response ability and multi-functionality of the nanohybrids and the wound caring compliance by the collagen patch. Meanwhile, the co-existence of these two components was necessary for fulfilling the orchestrated cascade, including identifying special pathological character in diabetic wound, triggered dualdrugs release in a close-loop manner and in situ hydrogel transformation. The collagen ark provided a framework for the nanohybrid to exert its designed functionality without causing unnecessary irritation, making it more suitable for wound caring. Meanwhile, the degradation product of the nanohybrid can prominently improve the mechanical property of the collagen patch. More importantly, the newly developed system showed a healing stage-specific proliferative enhancement. Hence, the tissue regeneration was biomimetically accelerated without any potent side effects. Both in vitro and in vivo results confirmed feasibility of the proposed cascade, and the superior treatment effect and better prognosis of this newly developed system compared to the free drugs. Overall, the results presented here support the development of a nanoparticle/ patch system for diabetic wound management, and potentially for other tissue engineering applications.

\section{Experimental}

Details on the experimental procedures are provided in the ESI. $\dagger$

\section{Conflicts of interest}

The authors declare no competing financial interests.

\section{Acknowledgements}

Z. Liu and Y. Li contributed equally to this work. Z. Liu acknowledges the Chinese Scholarship Council for financial support. Dr W. Li acknowledges the Orion Research Foundation for financial support. Prof. H. Zhang acknowledges Jane and Aatos Erkko Foundation (Decision No. 4704010), Academy of Finland (Grant No. 297580) and Sigrid Jusélius Foundation (Decision No. 28001830K1) for financial support. Prof. X. Deng acknowledges financial support from the Ministry of Science and Technology (Grants No. 2017YFA0504504 and 2016YFA0502001) and the National Natural Science Foundation of China (Grants No. 81422045, U1405223 and 81661138005), the Fundamental Research Funds for the Central Universities of China (Grant No. 20720160064), and the Program of Introducing Talents of Discipline to Universities (111 Project, B12001). Prof. H. A. Santos acknowledges financial support from the University of Helsinki Funds, the Sigrid Juselius Foundation (Decision No. 4704580), the HiLIFE Research Funds, and the European Research Council under the European Union's Seventh Framework Programme (FP/2007-2013, Grant No. 310892).

\section{Notes and references}

1 J. S. Mohammed and W. L. Murphy, Adv. Mater., 2010, 21, 2361-2374.

2 K. F. Bruggeman, R. J. Williams and D. R. Nisbet, $A d v$. Healthcare Mater., 2018, 7, 1700836.

3 A. Tian, B. Wang and J. Jiang, Proc. Natl. Acad. Sci. U. S. A., 2017, 114, E2699.

4 J. Yi, Y. Wang, Y. Jiang, I. Jung, W. Liu, V. Andrade, R. Xu, R. Parameswaran, I. Peters and R. Divan, Nat. Commun., 2017, 8, 509.

5 V. Falanga, Lancet, 2005, 366, 1736-1743.

6 R. J. Lee, M. L. Springer, W. E. Blanco-Bose, R. Shaw, P. C. Ursell and H. M. Blau, Circulation, 2000, 102, 898-901.

7 L. L. Wang and J. A. Burdick, Adv. Healthcare Mater., 2016, 6, 1601041.

8 A. Rege, N. V. Thakor, K. Rhie and A. P. Pathak, Angiogenesis, 2012, 15, 87-98.

9 H. Hashimoto and R. L. Prewitt, Int. J. Microcirc.: Clin. Exp., 1987, 5, 303.

10 S. A. Eming, B. Brachvogel, T. Odorisio and M. Koch, Prog. Histochem. Cytochem., 2008, 42, 115-170.

11 J. Martin, C. Nelson, M. Gupta, F. Yu, S. Sarett, K. Hocking, A. Pollins, L. Nanney, J. Davidson and S. Guelcher, Adv. Healthcare Mater., 2016, 5, 2751-2757.

12 D. Zhang, Y. Wei, K. Chen, X. Zhang, X. Xu, Q. Shi, S. Han, X. Chen, H. Gong and X. Li, Adv. Healthcare Mater., 2015, 4, 69-76.

13 K. Broaders, S. Grandhe and J. Fréchet, J. Am. Chem. Soc., 2010, 133, 756-758.

14 C. de Gracia Lux, S. Joshi-Barr, T. Nguyen, E. Mahmoud, E. Schopf, N. Fomina and A. Almutairi, J. Am. Chem. Soc., 2012, 134, 15758-15764.

15 R. M. Nzietchueng, B. Dousset, P. Franck, M. Benderdour, P. Nabet and K. Hess, J. Trace Elem. Med. Biol., 2002, 16, 239-244. 
16 S. Demirci, A. Doğan, S. Aydın, E. Ç. Dülger and F. Şahin, Mol. Cell. Biochem., 2016, 417, 119-133.

17 C. Song, F. Du and Z. Li, J. Mater. Chem. B, 2014, 2, 3413-3426.

18 C. Tapeinos and A. Pandit, Adv. Mater., 2016, 28, 5334.

19 V. Arul, J. G. Masilamoni, E. P. Jesudason, P. J. Jaji, M. Inayathullah, D. G. Dicky John, S. Vignesh and R. Jayakumar, J. Biomater. Appl., 2012, 26, 917.

20 M. M. Jr, Surg. Clin. North Am., 1984, 64, 769-778.

21 Z. Gu, A. Aimetti, Q. Wang, T. Dang, Y. Zhang, O. Veiseh, H. Cheng, R. S. Langer and D. G. Anderson, ACS Nano, 2013, 7, 4194-4201.

22 V. Ravaine, C. Ancla and B. Catargi, J. Controlled Release, 2008, 132, 2-11.

23 J. Wang, Y. Ye, J. Yu, A. R. Kahkoska, X. Zhang, C. Wang, W. Sun, R. D. Corder, Z. Chen and S. A. Khan, ACS Nano, 2018, 12, 2466-2473.

24 Y. Zhang, J. Wang, J. Yu, D. Wen, A. R. Kahkoska, Y. Lu, X. Zhang, J. B. Buse and Z. Gu, Small, 2018, 14, 1704181.

25 Z. Liu, Y. Li, W. Li, C. Xiao, D. Liu, C. Dong, M. Zhang, E. Mäkilä, M. Kemell and J. Salonen, Adv. Mater., 2018, 30, 1703393.

26 D. Liu, H. Zhang, E. Mäkilä, J. Fan, B. Herranz-Blanco, C. F. Wang, R. Rosa, A. J. Ribeiro, J. Salonen and J. Hirvonen, Biomaterials, 2015, 39, 249-259.

27 W. Li, Z. Liu, F. Fontana, Y. Ding, D. Liu, J. T. Hirvonen and H. A. Santos, Adv. Mater., 2018, 30, 1703740.

28 Z. Liu, V. Balasubramanian, C. Bhat, M. Vahermo, E. Mäkilä, M. Kemell, F. Fontana, A. Janoniene, V. Petrikaite and J. Salonen, Adv. Healthcare Mater., 2018, 6, 1601009.

29 L. M. Bimbo, M. Sarparanta, H. A. Santos, A. J. Airaksinen, E. Mäkilä, T. Laaksonen, L. Peltonen, V. P. Lehto, J. Hirvonen and J. Salonen, ACS Nano, 2010, 4, 3023-3032.

30 V. Grotheer, M. Goergens, P. C. Fuchs, S. Dunda, N. Pallua, J. Windolf and C. V. Suschek, Biomaterials, 2013, 34, 7314-7327.

31 S. Quignard, T. Coradin, J. J. Powell and R. Jugdaohsingh, Colloids Surf., B, 2017, 155, 530.

32 J. Hu, Y. Quan, Y. Lai, Z. Zheng, Z. Hu, X. Wang, T. Dai, Q. Zhang and Y. Cheng, J. Controlled Release, 2017, 247, 145.

33 Z. Wei, D. M. Lewis, Y. Xu and S. Gerecht, Adv. Healthcare Mater., 2017, 6, 1700523.

34 Y. Zhou, P. Damasceno, B. Somashekar, M. Engel, F. Tian, J. Zhu, R. Huang, K. Johnson, C. Mcintyre and K. Sun, Nat. Commun., 2018, 9, 54.

35 X. Sun, Q. Lang, H. Zhang, L. Cheng, Y. Zhang, G. Pan, X. Zhao, H. Yang, Y. Zhang and H. A. Santos, Adv. Funct. Mater., 2016, 27, 1604617.

36 S. L. Lu, L. Qiao, T. Xie, Y. Yang, S. Jin and C. Qing, Natl. Med. J. China, 2005, 85, 1899-1902.

37 X. Geng, X. Mo, L. Fan, A. Yin and J. Fang, J. Mater. Chem., 2012, 22, 25130-25139.

38 D. Liu, H. Zhang, E. Mäkilä, J. Fan, B. Herranz-Blanco, C. Wang, R. Rosa, A. J. Ribeiro, J. Salonen and J. Hirvonen, Biomaterials, 2015, 39, 249-259.
39 I. Ydens, D. Rutot, P. Degée, J. Six, E. Dellacherie and P. Dubois, Macromolecules, 2000, 33, 6713-6721.

40 S. Yang, Z. Tang, D. Zhang, M. Deng and X. Chen, Biomater. Sci., 2017, 5, 2169-2178.

41 T. Jiao, J. Zhou, J. Zhou, L. Gao, Y. Xing and X. Li, Iran. Polym. J., 2011, 20, 123-136.

42 X. Hu, J. Yu, C. Qian, L. Yue, A. R. Kahkoska, Z. Xie, X. Jing, J. B. Buse and G. Zhen, ACS Nano, 2017, 11, 613.

43 M. Sheikholeslam, W. Mee, M. G. Jeschke and S. Amini-Nik, Adv. Healthcare Mater., 2018, 7, 1700897.

44 X. Zhang, Y. Yang, J. Yao, Z. Shao and X. Chen, ACS Sustainable Chem. Eng., 2014, 2, 1318-1324.

45 R. Attalla, C. Ling and P. R. Selvaganapathy, Adv. Healthcare Mater., 2018, 7, 1701385.

46 Y. Zhou, P. F. Damasceno, B. S. Somashekar, M. Engel, F. Tian, J. Zhu, R. Huang, K. Johnson, C. Mcintyre and K. Sun, Nat. Commun., 2018, 9, 54.

47 W. Friess, G. Lee and M. J. Groves, Pharm. Dev. Technol., 1996, 1, 185-193.

48 D. Duscher, E. Neofytou, V. W. Wong, Z. N. Maan, R. C. Rennert, M. Inayathullah, M. Januszyk, M. Rodrigues, A. V. Malkovskiy and A. J. Whitmore, Proc. Natl. Acad. Sci. U. S. A., 2015, 112, 94-99.

49 H. Chen, P. Jia, H. Kang, H. Zhang, Y. Liu, P. Yang, Y. Yan, G. Zuo, L. Guo and M. Jiang, Adv. Healthcare Mater., 2016, 5, 907-918.

50 S. Farsaei, H. Khalili and E. S. Farboud, Int. Wound J., 2012, 9, 238-247.

51 J. J. Akershoek, K. M. Brouwer, M. Vlig, B. Bkhl, B. Rhj, E. Middelkoop and U. Mmw, PLoS One, 2017, 12, e0179350.

52 M. Tahvanainen, T. Rotko, E. Mäkilä, H. A. Santos, D. Neves,

T. Laaksonen, A. Kallonen, K. Hämäläinen, M. Peura and R. Serimaa, Int. J. Pharm., 2012, 422, 125.

53 G. F. Pierce, Am. J. Physiol., 2001, 159, 399-403.

54 V. Kant, D. Kumar, D. Kumar, R. Prasad, A. Gopal, N. N. Pathak, P. Kumar and S. K. Tandan, Cytokine, 2015, 73, 144-155.

55 I. R. Botusan, V. G. Sunkari, O. Savu, A. I. Catrina, J. Grünler, S. Lindberg, T. Pereira, S. Ylä-Herttuala, L. Poellinger and K. Brismar, Proc. Natl. Acad. Sci. U. S. A., 2008, 105, 19426-19431.

56 H. Karvinen, E. Pasanen, T. T. Rissanen, P. Korpisalo, E. Vähäkangas, A. Jazwa, M. Giacca and S. Yläherttuala, Gene Ther., 2011, 18, 1166-1172.

57 P. Carmeliet, Nat. Med., 2000, 6, 1102-1103.

58 T. A. Markel, P. R. Crisostomo, M. Wang, C. M. Herring, T. Lahm, K. K. Meldrum, K. D. Lillemoe, F. J. Rescorla and D. R. Meldrum, Am. J. Physiol., 2007, 292, G958-G963.

59 C. Ritter, A. A. Cunha, I. C. Echer, M. Andrades, A. Reinke, N. Lucchiari, J. Rocha, E. L. Streck, S. Menna-Barreto and J. C. Moreira, Crit. Care Med., 2006, 34, 471-477.

60 H. Chun, D. Kim, H. Yi, Y. Kim, E. Kim, S. Hwang, C. Jwa, Y. Lee and H. Ryou, Neurol. Sci., 2012, 33, 289. 